\title{
GENERATION OF KILOVOLT, PICOSECOND ELECTRIC PULSES BY COHERENT COMBINING IN OPTOELECTRONIC SYSTEM
}

\author{
Sahar Wehbi ${ }^{\mathrm{a}, \mathrm{b}}$, Farid El Bassri ${ }^{\mathrm{a}}$, Dominique Pagnoux ${ }^{\mathrm{a}}$, Philippe Leproux ${ }^{\mathrm{a}}$, Delia Arnaud-Cormos ${ }^{\mathrm{a}, \mathrm{c}}$, \\ Philippe Leveque ${ }^{\mathrm{a}}$ Anthony Bertrand ${ }^{\mathrm{b}}$ and Vincent Couderc ${ }^{\mathrm{a}}$ \\ aUniv. Limoges, XLIM, UMR CNRS 7252, 123 av. A. Thomas, 87060 Limoges, France \\ ${ }^{\mathrm{b}} \mathrm{ALPhANOV}$, Centre technologique optique et lasers, Institut d'optique d'Aquitaine, Rue François \\ Mitterrand, 33400 TALENCE \\ ${ }^{\mathrm{C}}$ Institut Universitaire de France (IUF), 1 rue Descartes, 75005 Paris, France
}

\begin{abstract}
Nowadays, interactions between pulsed electromagnetic field and biological cells and tissues are particularly investigated to prevent any noxious effects and/or to develop therapeutic processes able to improve cancer treatment. Electrochemotherapy is an example which allows drugs delivery improvement thanks to local pulsed electric field application. Consequently, high voltage electrical nanosecond pulse generation has received great interest from the researchers working in the bioelectromagnetic domain. In that framework, we demonstrated that picosecond kilovolt generator activated by a nanosecond and femtosecond laser sources can be coupled with a Multiplex Coherent AntiStokes Raman Scattering system (M-CARS) to study the impact of electric pulses on biological cells. We generated kilovolt picosecond electric pulses by using a frozen-wave generator which integrated silicon PhotoConductive Semiconductors Switches (PCSSs). Because of the linear switching regime no temporal jitter is observed during the optical switching thus, coherent combining of short electric pulses can be obtained. Two semiconductors are activated with adjustable time delay, generating unipolar pulses with less than 60 ps rise time (pulse duration 100 ps). Balanced and unbalanced bipolar pulses have been also obtained with a peak to peak voltage of $1.4 \mathrm{kV}$ and a total duration of 244 ps. The initial optical pulse may be used to produce a supercontinnum extended from the visible and up to infrared domain $(2.4 \mu \mathrm{m})$. Thus synchronized M-CARS diagnosis can be realized when nanosecond and picosecond electric pulse excitation is applied to biological samples.
\end{abstract}

Keywords: Optoelectronic switching, Picosecond electric pulse generation, Linear switching regime, EM-CARS experiment, Frozen-wave generator.

\section{INTRODUCTION}

It is well known that microsecond and nanosecond electric pulses may modify the cell membrane permeability and thus enhance drug delivery into cells $[1,2]$. However, this mechanism depends on the shape of the electric pulses and mainly of the high frequencies which compose the pulse spectrum. If nanosecond electric pulses have a direct effect on the cell plasma membrane, high frequencies can more deeply affect cell organelles and penetrate further in nucleus and mitochondrias [3]. It is also important to underline that picosecond pulses are shorter than the plasma membrane charging time constant and thus, one can speculate that permeabilisation with picosecond pulses could be different from with nanosecond pulses [4]. Indeed, if charges displacement seems to be the dominant effect with long pulses, ultrafast nonlinear field effects may appear in organelles and significantly disrupt cell metabolism mixing fast physical effects. In these conditions the main difficulty is to quantify and separate the physical phenomena appearing with a short response time from the biological one more clearly visible at longer time scale.

In this context, we developed a hybrid Electro-Multiplex Coherent Anti-Stokes Raman Scattering system (EM-CARS), able to produce, kilovolt nanosecond and picosecond electric pulses without timing jitter on one hand and capable to investigate any change in the vibrational signature of cells on the other hand,. That electric-optic pump/probe system is controlled by a single femtosecond/picosecond (350 fs - $10 \mathrm{ps)} \mathrm{powerful} \mathrm{laser} \mathrm{source} \mathrm{that} \mathrm{feeds} \mathrm{the} \mathrm{optoelectronic}$ generator and the M-CARS system. Because of the use of PCSS technology in linear switching regime, we successfully 
controlled the temporal and spectral shapes of the electric pulses (rise time down to $50 \mathrm{ps,} \mathrm{pulse} \mathrm{duration} 100 \mathrm{ps)}$ ). The "frozen wave generator" that we used here is activated by optical femtosecond pulses with adjustable energy/delay and allows coherent sum of electric pulses activated in turn by two PCSSs. Thus, unipolar and bipolar kilovolt pulses with adjustable shape, in real time, have been produced [5]. The M-CARS system is based on the same optical pulse source where the broadband Stokes beam is generated in single mode and in multimode optical GRIN fibers with Kerr selfcleaning effect.

\section{METHODOLOGY}

The pulse generator used in this work is based on the frozen wave generator principle. Figure 1 shows the experimental setup. It is composed of, at least, two photoconductive semiconductors switches (PCSSs), separated by a $50 \Omega$ transmission line. The first "PCSS1" is connected to a 70-GHz spectral bandwidth oscilloscope with four attenuators, while the second "PCSS2" is connected to the ground. These PCSSs are made with silicon semiconductors and can sustain up to $4 \mathrm{kV}$ in $\mathrm{CW}$ configuration. A high voltage source is added to supply the central transmission line during the off-state. A femtosecond laser source (Laser Tangerine HP) with $1030 \mathrm{~nm}$ wavelength is used to activate the system. This source provides optical pulses down to $350 \mathrm{fs}$ with tunable repetition rate $(1 \mathrm{~Hz}-40 \mathrm{MHz})$ and a pulse energy up to $200 \mu \mathrm{J}$. Because of the linear mode excitation it is possible to control the temporal features of the generated picosecond electric pulses. The shape of the output pulses is related to the length of the central line and to the switching conditions of the PCSSs. The delay between optical excitations of the two PCSSs and the modulation of the optical energy may provide many different conditions to control the shape of the electric pulses (unipolar, balanced bipolar, unbalanced bipolar...).

(a)

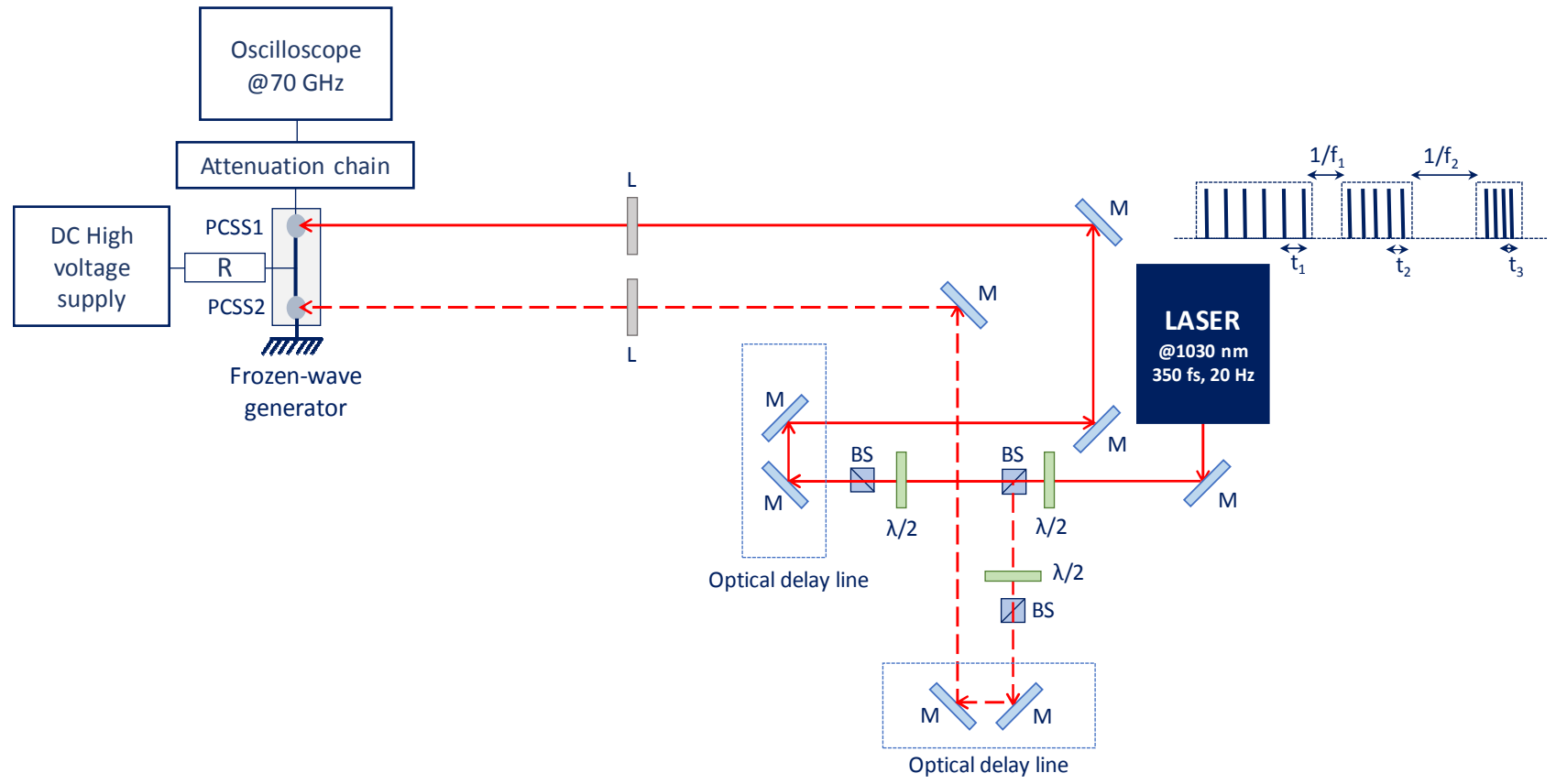

Figure 1. (a) Experimental setup for the generation of picosecond electric pulses with adjustable temporal profiles. Legend: M: mirror; $\lambda / 2$ : half-wave plate; BS: beam splitter L: lens; PCSS: photoconductive semi-conductor switch, R: resistor; (b) Optical pulses with variable duration and frequency generated by femtosecond laser @1030nm. 


\section{RESULTS}

\subsection{Generation of picosecond and nanosecond bipolar pulses}

\section{Picosecond pulses}

In this section, the possibility of ultrashort electric pulse generation (picosecond domain) with adjustable shapes has been investigated using the "Frozen wave generator" configuration. A transmission line of $2 \mathrm{~mm}$ length has been chosen to connect the two PCSSs. For an input voltage of $4 \mathrm{kV}$, the two switches were excited simultaneously by the femtosecond optical pulses. A bipolar pulse has been obtained having a total duration of 244 ps with a central fall time of 40 ps (Figure 2(a)). The created output voltage in this case was equal to $1.4 \mathrm{kV}$. The spectrum of the bipolar output pulse covers more than $9.5 \mathrm{GHz} @-20 \mathrm{~dB}$ (Figure 2(b)). That output pulse is the result of the coherent combining of two different pulses obtained separately when switching either PCSS1 or PCSS2. For a single activation of PCSS1 a unipolar positive pulse was obtained (Figure 2 (c)). The pulse duration was then close to $140 \mathrm{ps}$. On the contrary, the illumination of the second switch (PCSS2) alone generated an unbalanced negative pulse exhibiting 100 ps duration for the positive part and 100 ps duration for the negative one (Figure 2(d)).

(a)

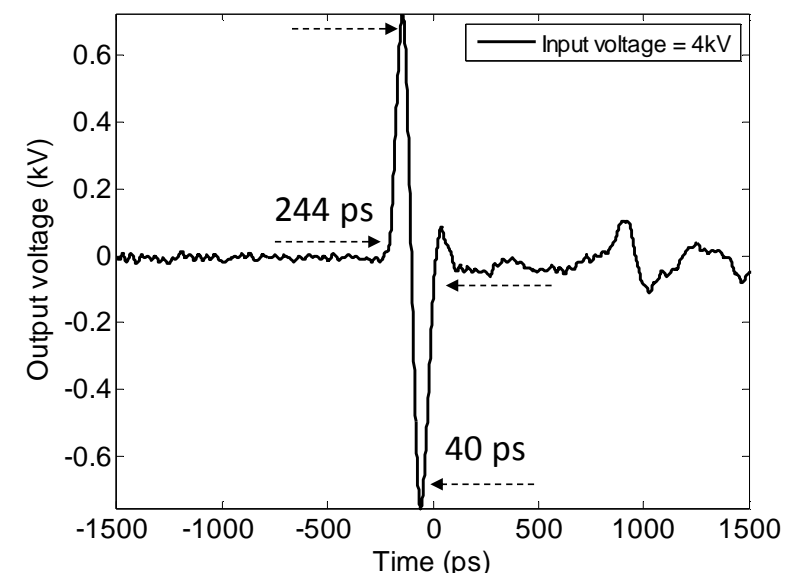

(c)

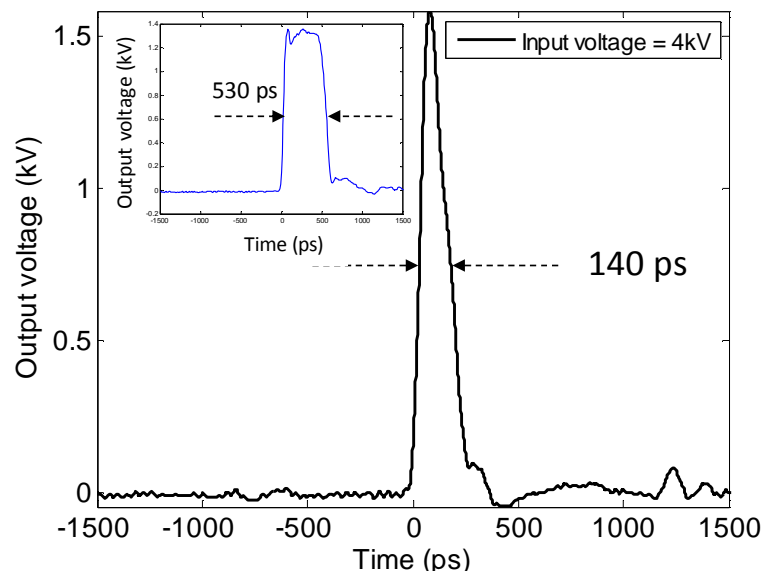

(b)

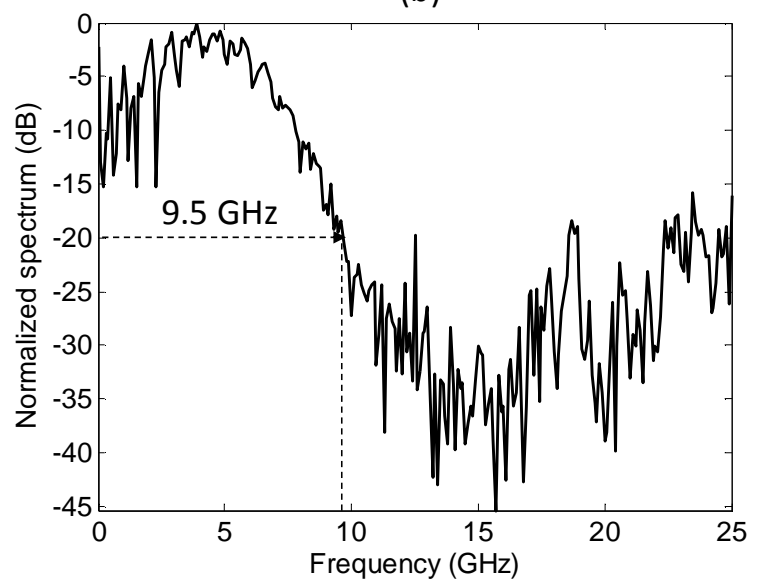

(d)

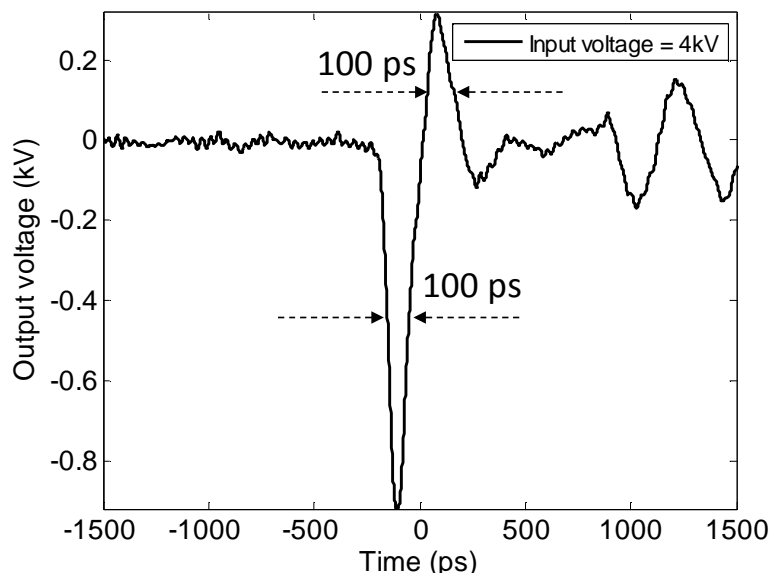

Figure 2. (a) Balanced bipolar pulse resulting from the coherent pulse combining when PCSS1 and PCSS2 are activated with a given delay; (b) spectrum of the bipolar electric pulse; (c) Unipolar positive pulse generated when only PCSS1 is illuminated, (d) negative pulse generation when only PCSS2 is illuminated. Inset figure (c): example of long unipolar output pulse obtained with femtosecond excitation. 
By illuminating with only few $\mu \mathrm{J}(3 \mu \mathrm{J})$ on the PCSS2 it was possible to control the output impedance of the PCSS2 and to fix it at $50 \Omega$. In that configuration a unipolar output pulse has been obtained with adjustable duration by managing the switching delay between PCSS1 and PCSS2. Picosecond pulses from 100 ps and up to the nanosecond scale have been obtained with more than $2 \mathrm{kV}$ of peak voltage. An example of output unipolar long pulse is shown in the inset of figure 2(c).

The control of the relative excitation delay between the two PCSSs and the management of the energy lighting the semiconductors may permit to shape the bipolar pulse by reducing or amplifying its negative part of the output pulse (see Figure 3). Thus the spectral profile carried by the pulse evolves from tens of $\mathrm{MHz}$ and up to $\mathrm{GHz}$ domains.

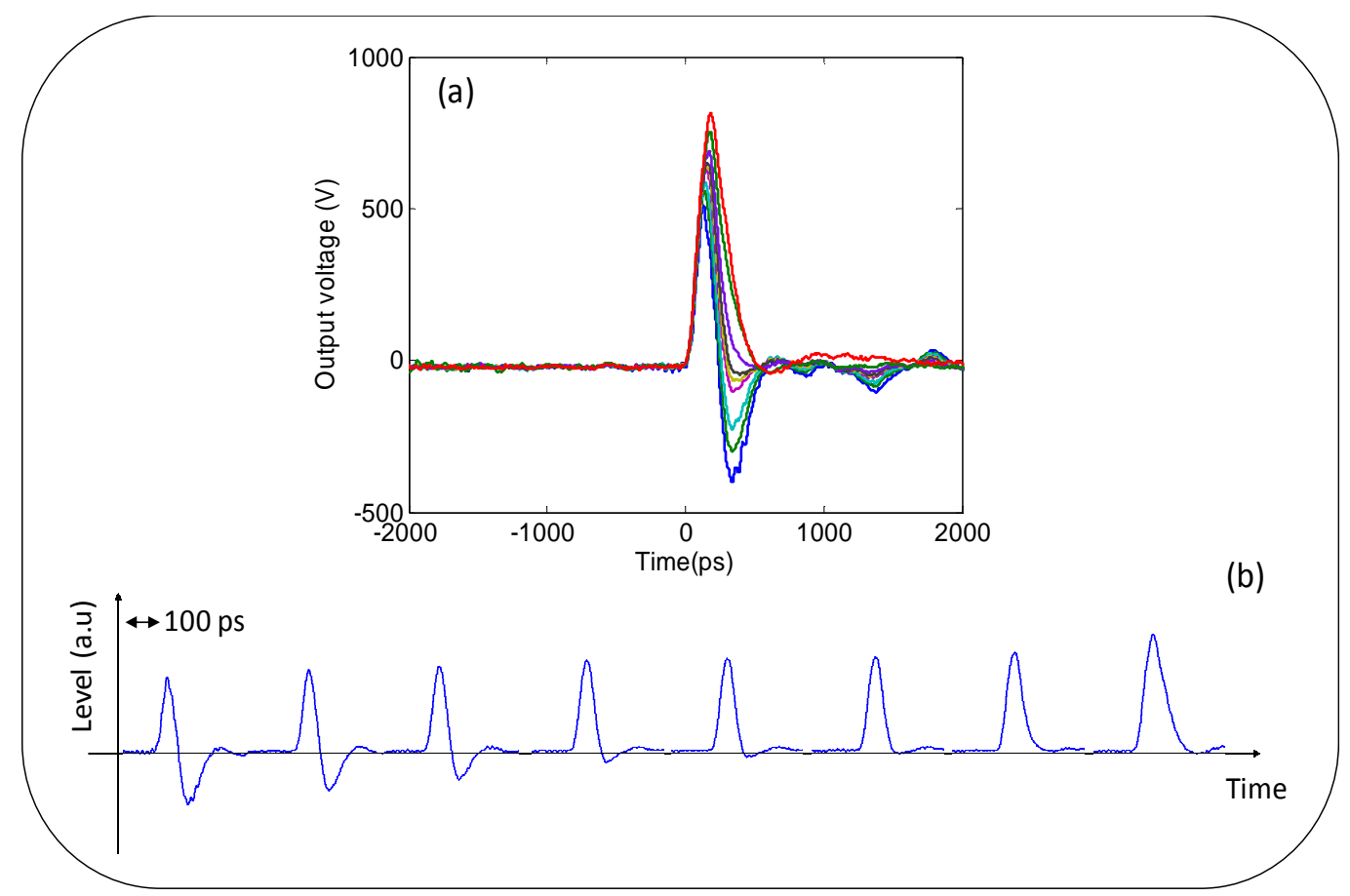

Figure 3. (a) Bipolar pulse evolution versus the time delay of excitation between the two PCSSs combined with the energy carried by the two optical pulses. (a) Output pulse evolution, (b) Snapshots reproducing the temporal reshaping.

\section{Nanosecond pulses}

The same generation can be realized in nanosecond scale to improve the output energy carried by the electrical pulses. This is achieved by extending the length of the propagating line located between the two PCSSs and by using a $2 \mathrm{~ns}$ laser source with millijoule optical pulse at $1064 \mathrm{~nm}$. One result of this experiment is shown on the figure 4 . For a $30 \mathrm{~cm}$ length of the central line, a balanced bipolar pulse of $23 \mathrm{~ns}$ is obtained (see figure 4(a)). The corresponding output spectrum bandwidth is then close to $100 \mathrm{MHz}$. In that configuration, an electric excitation of cells with ns pulses will mainly produce permeabilization of the plasma membrane. An additional spectral shaping may be obtained by exciting only the first PCSS or by delaying the activation of the second PCSS. In that last case, two output electrical pulses are generated with adjustable delay between them (see figure 4(c) and 4(d)). 
(a)

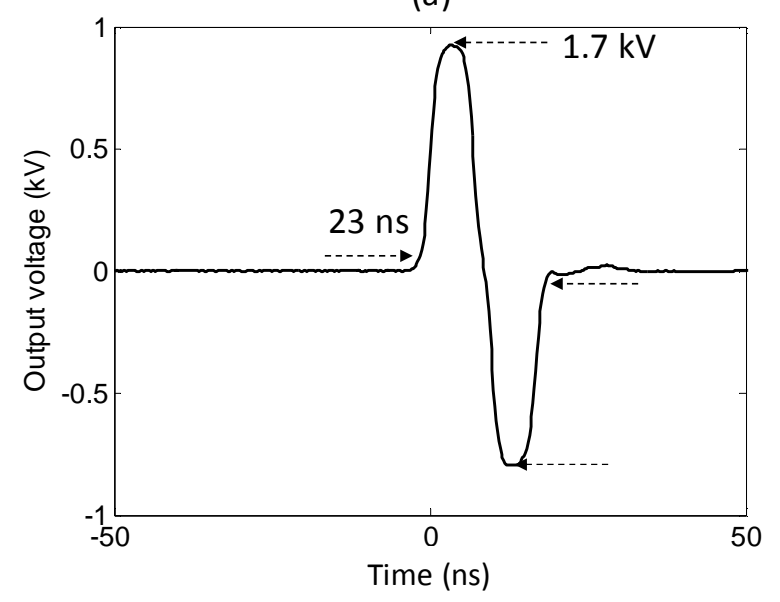

(c)

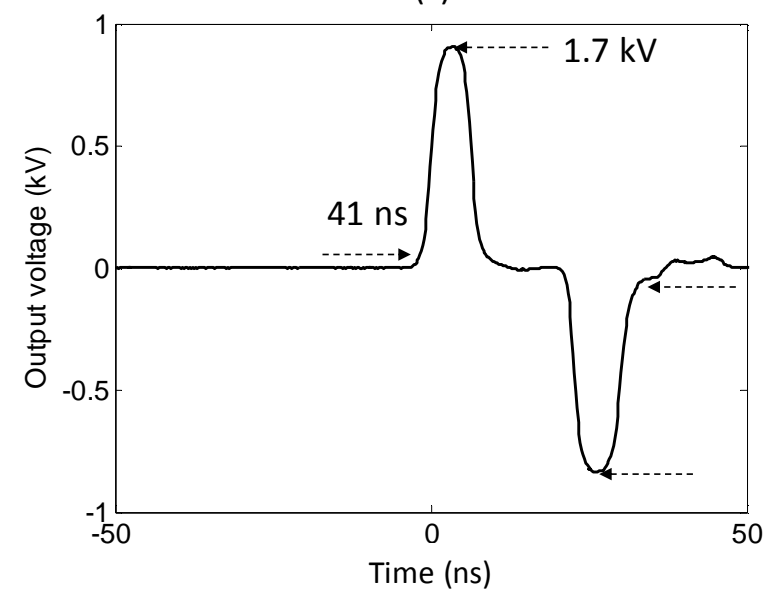

(b)

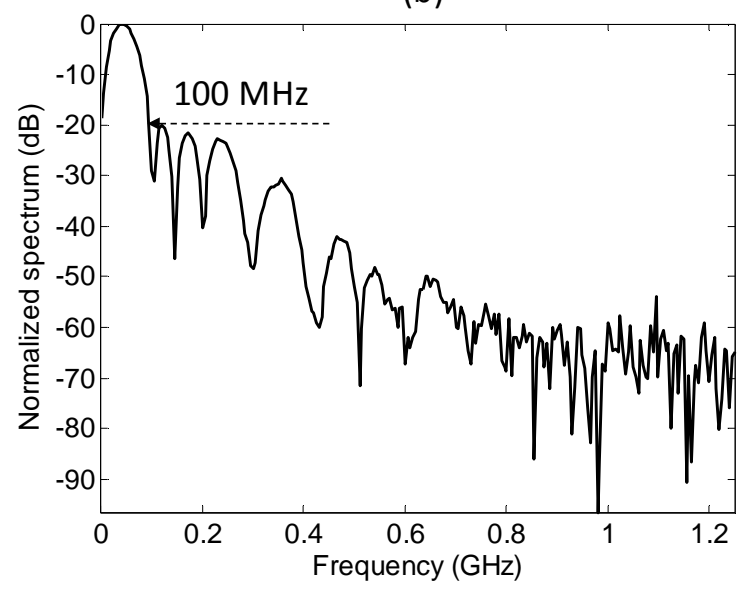

(d)

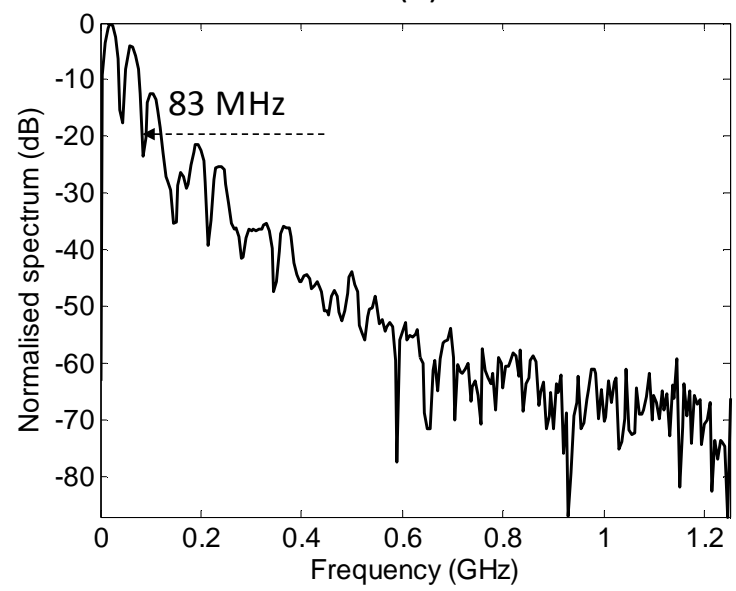

Figure 4. (a) balanced bipolar pulse obtained in nanosecond regime, (b) output spectrum of the bipolar pulse, (c) bipolar pulse generation with adjustable delay between the positive and negative components, (d) output spectrum of the delayed bipolar pulses.

\subsection{Optical energy impact on the output pulse duration}

Beyond the pulse shaping demonstration we also studied the effect of the optical energy on the switching dynamics [6]. Accordingly, a single photoconductive semi-conductor switch has been connected to the oscilloscope on one side, and to the high voltage power supply by means of a resistor $(100 \mathrm{k} \Omega)$ on the other side. For an input voltage of $4 \mathrm{kV}$, the diode has been excited by different optical energies extending from $5 \mu \mathrm{J}$ to $55 \mu \mathrm{J}$. Thus, the variation of the output voltage, of the pulse duration (Full Width at Half of the Maximum "FWHM"), of the switching efficiency and rise time were measured. It can be noticed that the maximum output voltage was only $1.64 \mathrm{kV}$ because of internal losses inside the frozen wave generator activated by short optical pulses (350 fs). The optimal incident input energy was $20 \mu \mathrm{J}$ (see figure $5(\mathrm{a})$ ). This corresponds to a maximum of switching efficiency close to $82 \%$. For higher optical energy, space charge appears inside the semiconductor and limits the output current. Therfore, switching efficiency falls down to $70 \%$ (see figure 6 - blue curve). 
(a)

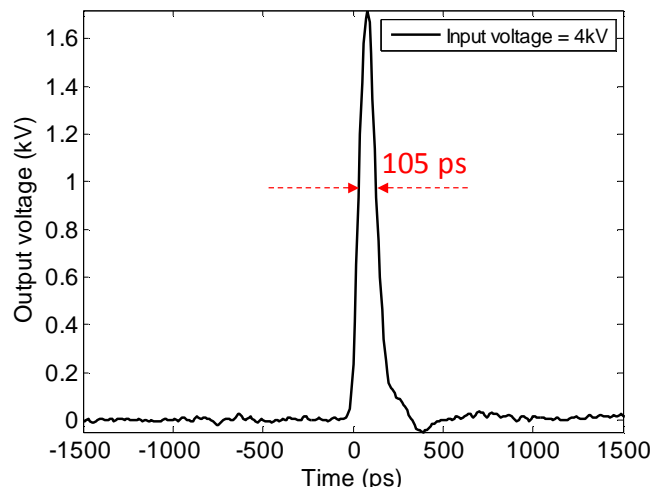

(b)

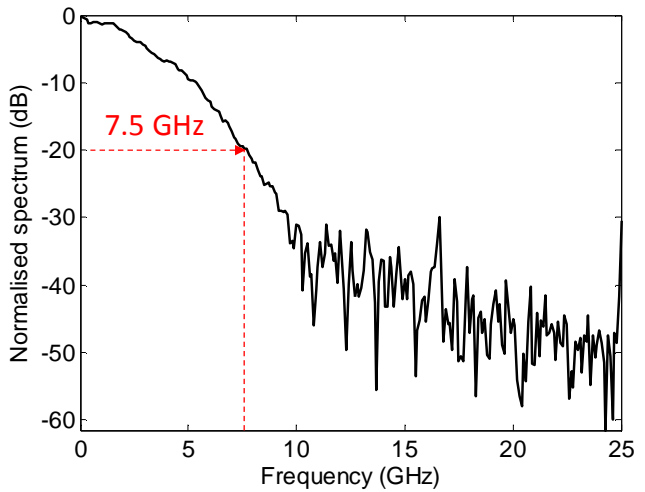

Figure 5. Unipolar positive electric pulse shape $(20 \mu \mathrm{J})$, (b) corresponding spectrum

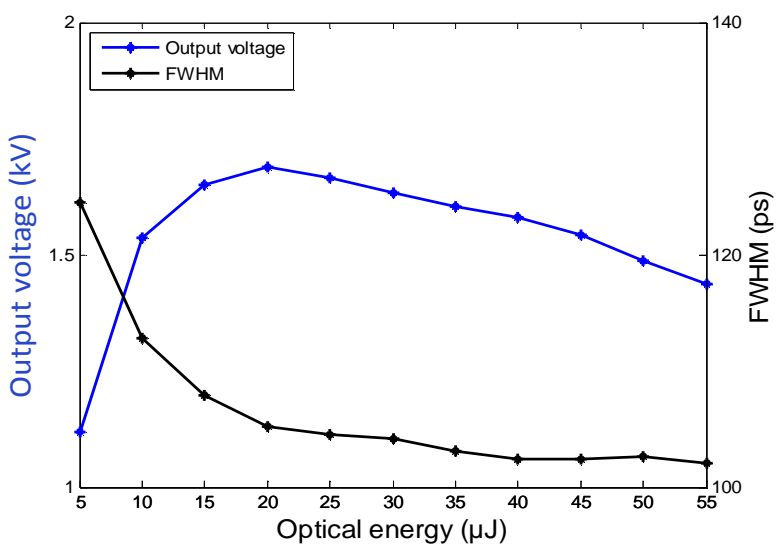

Figure 6. Output pulse voltage and duration versus the optical energy sent on the PCSS1, bias voltage: $4 \mathrm{kV}$.

However, the pulse rise time remains relatively constant close to 62 ps during the whole experimental conditions (see figure 7 (b)). This observation confirms that the used process corresponds to the linear switching regime where only the optical pulse features and the impedance of the generator drive the pulse characteristics in the time domain. It is also important to note that the increase of the optical pulse duration up to $10 \mathrm{ps}$ does not significantly improve the switching efficiency.

(a)

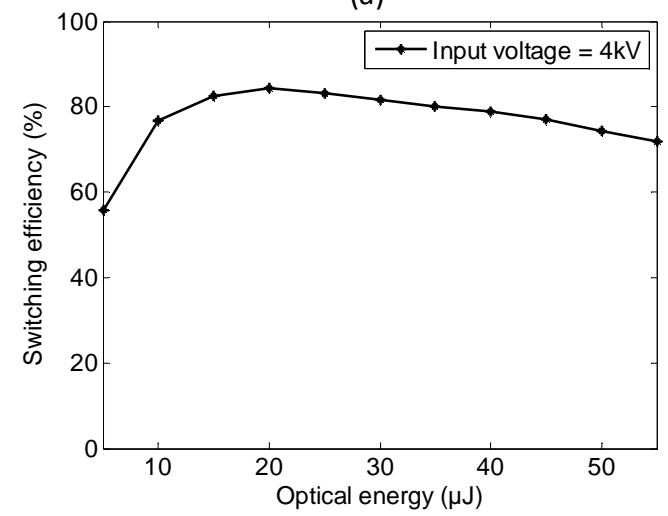

(b)

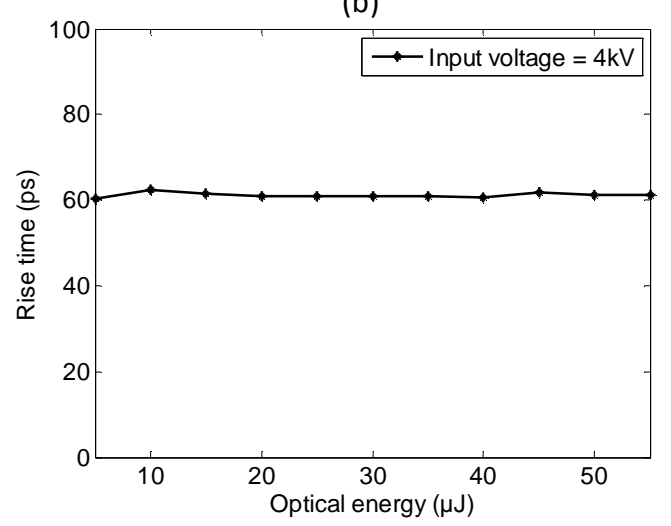

Figure 7. (a) Switching efficiency and (b) rise time versus the optical pulse energy for $4 \mathrm{kV}$ bias voltage. 


\subsection{Bias voltage impact on the output pulse shape}

The impact of the bias voltage on the electric pulse shape has been also investigated. Two PCSSs were connected in series. In that configuration the equivalent length of the central line constituting the frozen wave generator is increased in length with respect to the first one realized by using a single PCSS. Then longer electric pulses are expected. The PCSSs were connected to the oscilloscope on one side and to the high voltage power supply on the other side. It has been possible to increase the bias voltage beyond $4 \mathrm{kV}$ by using two switches arranged in series. The excitation of the two PCSSs have been synchronized. Thus, unipolar picosecond electric pulse was generated up to $6 \mathrm{kV}$ of bias voltage. The maximum output voltage was $2.3 \mathrm{kV}$ which represented a switching efficiency of $76 \%$. The pulse duration started from $158 \mathrm{ps}$ and increased with the input voltage up to $180 \mathrm{ps}$ (see figure 8). Similarly the pulse rise time evolved from 60 to $80 \mathrm{ps}$ between 1 and $6 \mathrm{kV}$. The linear evolution of the output voltage indicates also the absence of Keldish effect during the switching dynamics which remains stable close to $76 \%$ (see figure 8 ).

(a)

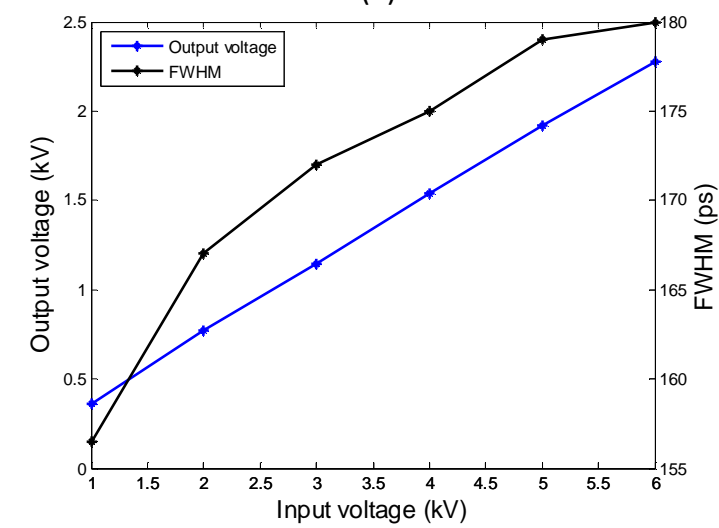

(c)

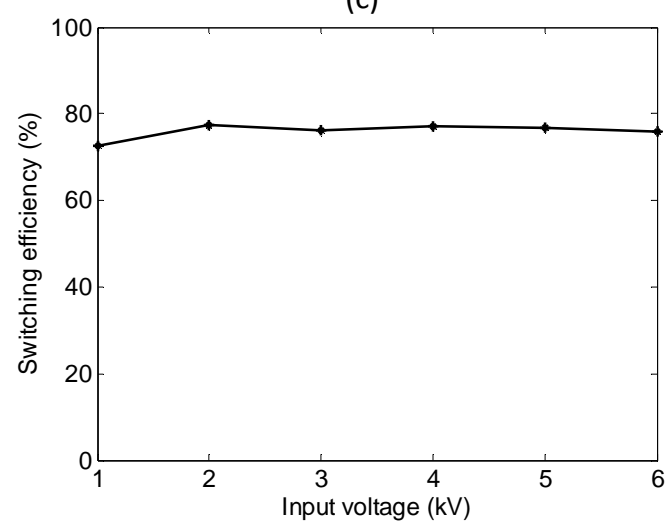

(b)

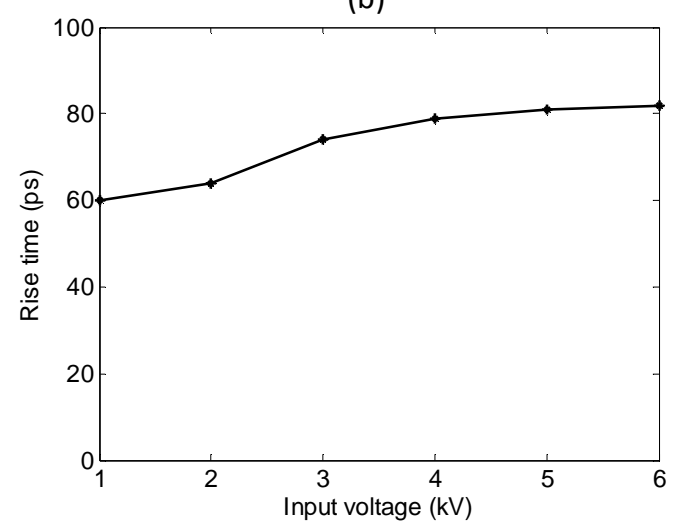

Figure 8. (a) Output voltage and pulse duration, (b) Switching efficiency, (c) Pulse rise time versus the bias voltage.

\section{ELECTRO-CARS EXPERIMENT IN PULSED REGIME}

The experimental setup used for electro-CARS experiment is shown on the figure 9. It is constituted of a pulsed laser source at $1064 \mathrm{~nm}(30 \mathrm{kHz})$, a switching system similar to the one described previously in that paper, a CARS setup integrating a pump and a broadband Stokes wave and a microscope coupled to a high resolution spectroscope $\left(0.36 \mathrm{~cm}^{-}\right.$ ${ }^{1}$ ). The optoelectronic generator presented previously is particularly well suited for that pump-probe experiment and provides electrical pulses perfectly synchronized with the optical pump and Stokes waves used in the CARS 
spectroscopy. The measured temporal jitter between electrical and optical pulses is lower than $1 \mathrm{ps} \mathrm{[7-11].} \mathrm{This} \mathrm{perfect}$ temporal synchronization is driven by the optoelectronic generation running in the linear switching regime [8].

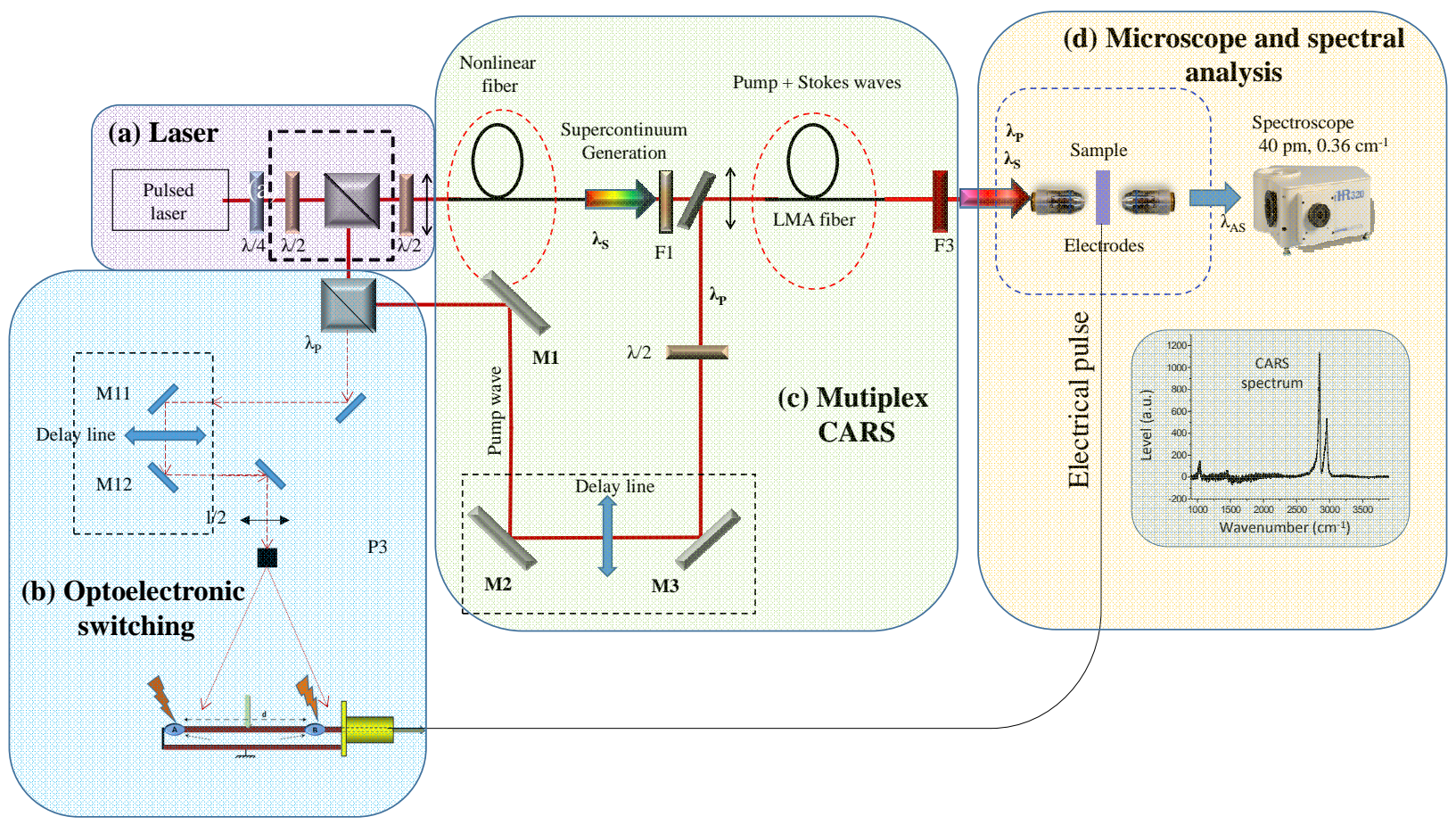

Figure 9. Experimental setup of the EM-CARS, (a) Laser source, (b) Optoelectronic generator, (c) CARS system composed of a pump and a large band Stokes wave [8], (d) Microscope and spectroscope for EM- CARS experiment.

By using this experimental setup we obtained a CARS signal from 500 to $3500 \mathrm{~cm}^{-1}$ (see inset figure 9(d)).The first electrical pulses have been generated in the nanosecond scale with a positive square shape of $2.5 \mathrm{~ns}$, peak voltage higher than $1.6 \mathrm{kV}$.

We focused our attention on a dimethyl sulfoxide sample which is an organosulfur compound (DMSO). We first performed the CARS experiment without electric stimulation. We recorded the spectral signature of the DMSO in the $\mathrm{CH}$ region and we identified the symmetrical stretching vibration at $2920 \mathrm{~cm}^{-1}$. In a second step we add the electric stimulation with a polarization direction parallel to the optical polarization of the pump and Stokes waves. The EMCARS signal is integrated over one second. Thus EM-CARS spectra were recorded for peak voltages ranging from 0 to $1.4 \mathrm{kV}$ (Bias voltage of the frozen wave generator: $\sim 3 \mathrm{kV}$ ).

In that configuration, the wavelength of the EM-CARS peak resulting from the symmetrical stretching vibration (2920 $\mathrm{cm}^{-1}$ ) undergoes a shift towards the lowest wavenumbers when the input peak voltage of the nanosecond pulses increased. A maximum shift of $4 \mathrm{~cm}^{-1}$ (from $2922.4 \mathrm{~cm}^{-1}$ to $2918.4 \mathrm{~cm}^{-1}$ ) is obtained for a peak voltage of $1.4 \mathrm{kV}$. This shift is similar on the asymmetric $\mathrm{CH}_{3}$ peak appearing close to $3008 \mathrm{~cm}^{-1}$ (Figure 9). This spectral evolution is not linear with the peak voltage but dramatically increase for an electrical polarization higher than $0.8 \mathrm{kV}$. That sudden transition is due to the ionization of the DMSO leading to a local temperature increase. This first experimentation using a dynamic polarization of a DMSO sample by nanosecond electric pulses demonstrated the potentiality to detect the impact of a short electromagnetic wave (nanosecond) on a chemical compound. 

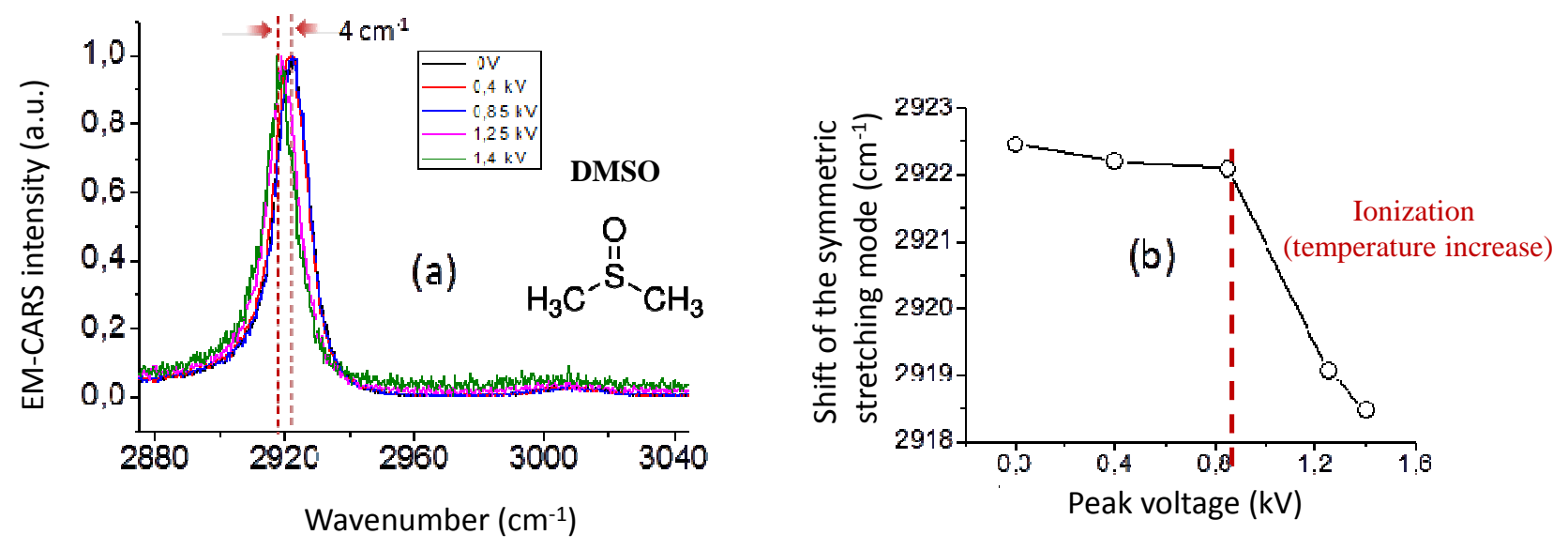

Figure 10. (a) EM-CARS spectrum versus peak voltage of electrical pulse (4.2 ns). (b) Shift of the symmetric stretching mode versus pulse peak voltage.

\section{CONCLUSION}

We demonstrated ultrashort electric pulses generation with adjustable temporal shapes by using a frozen-wave generator with a femtosecond laser source able to activate photoconductive semiconductor switches, in linear commutation regime. Because of the low temporal jitter, coherent combining of several picosecond electric pulses has been obtained by using temporal delay between each optical switching. Unipolar and bipolar pulses with less than $65 \mathrm{ps}$ and $100 \mathrm{ps}$ rise time and duration respectively have been demonstrated. The output shape of the pulse is adjustable in time and can exhibits up to $8 \mathrm{GHz} @-20 \mathrm{~dB}$ of spectrum bandwidth. The maximum peak voltage obtained with a bias voltage of $4 \mathrm{kV}$ was $1.6 \mathrm{kV}$. That optoelectronic generator can be coupled with a M-CARS imaging system able to investigate the impact of pulsed electric field on the molecular structure of biological samples. Evidences of structure destabilization on DMSO have been demonstrated with 4 ns pulses.

\section{REFERENCES}

[1] Beebe, S. J., Fox, P. M., Rec, L. J., Willis, E. L. K., and Schoenbach, K. H., "Nanosecond, high-intensity pulsed electric fields induce apoptosis in human cells," The FASEB journal, 17(11), 1493-1495 (2003).

[2] Capitaine, E., Moussa, N. O., Louot, C., Lefort, C., Pagnoux, D., Duclère, J. R., Kaneyasu, J. F., Kano, H., Duponchel, L., Couderc, V., and Leproux, P., "Coherent anti-Stokes Raman scattering under electric field stimulation," Physical Review B, 94(24), 245136 (2016).

[3] Schoenbach, K. H., Xiao, S., Camp, J. T., Migliaccio, M., Beebe, S. J., and Baum, C. E., "Wideband, highamplitude, pulsed antennas for medical therapies and medical imaging," In 2009 International Conference on Electromagnetics in Advanced (2009).

[4] Semenov, I., and Xiao, S., [Handbook of Electroporation], Springer International Publishing, 171-186 (2017).

[5] El Amari, S., Kenaan, M., Merla, C., Vergne, B., Arnaud-Cormos, D., Leveque, P., and Couderc, V., "Kilovolt, nanosecond, and picosecond electric pulse shaping by using optoelectronic switching," IEEE Photonics Technology Letters, 22(21), 1577-1579 (2010).

[6] El Amari, S., De Angelis, A., Arnaud-Cormos, D., Couderc, V., andLeveque, P., "Characterization of a linear photoconductive switch used in nanosecond pulsed electric field generator," IEEE Photonics Technology Letters, 23(11), 673-675 (2011). 
[7] Vergne, B., Couderc, V., and Leveque, P., “A 30-kHz monocycle generator using linear photoconductive switches and a microchip laser," IEEE Photonics Technology Letters, 20(24), 2132-2134 (2008).

[8] Vergne, B., Couderc, V., Barthélémy, A., Gontier, D., Lalande, M., andBertrand, V., "High-voltage rectifier diodes used as photoconductive device for microwave pulse generation," IEEE transactions on plasma science, 34(5), 1806-1813 (2006).

[9] Arnaud-Cormos, D., Couderc, V. and Leveque, P., [Handbook of Electroporation], Springer International Publishing, 1-21 (2017).

[10] Kohler, S., Couderc, V., O'Connor, R. P., Arnaud-Cormos, D., and Leveque, P., "A versatile high voltage nano-and sub-nanosecond pulse generator,". IEEE Transactions on Dielectrics and Electrical Insulation, 20(4), 1201-1208 (2013).

[11] Arnaud-Cormos, D., Couderc, V., Leveque, P., [Handbook of Electroporation], Springer International Publishing, 813-836 (2017). 